AperTO - Archivio Istituzionale Open Access dell'Università di Torino

\title{
BALB-neuT Female Mice as a Dynamic Model of Mammary Cancer
}

\section{This is a pre print version of the following article:}

Original Citation:

\section{Availability:}

This version is available http://hdl.handle.net/2318/129922

since 2017-05-17T14:10:34Z

Publisher:

Bentham Science Publishers

Published version:

DOI:10.2174/978160805469511201010139

Terms of use:

Open Access

Anyone can freely access the full text of works made available as "Open Access". Works made available under a Creative Commons license can be used according to the terms and conditions of said license. Use of all other works requires consent of the right holder (author or publisher) if not exempted from copyright protection by the applicable law. 
This is the author's final version of the contribution published as:

Iezzi M; Calogero RA; Spadaro M; Musiani P; Forni G; Cavallo F. BALB-neuT Female Mice as a Dynamic Model of Mammary Cancer. Bentham Science Publishers. 2012. pp: 139-166.

in

Translational Animal Models in Drug Discovery and Development

The publisher's version is available at:

When citing, please refer to the published version.

Link to this full text:

http://hdl.handle.net/2318/129922 


\title{
BALB-neuT female mice as a dynamic model of mammary cancer
}

\author{
Manuela Iezzi ${ }^{1}$, Raffaele A. Calogero ${ }^{2}$, Michela Spadaro ${ }^{2}$, Piero Musiani ${ }^{1}$, Guido Forni $^{2}$ and \\ Federica Cavallo ${ }^{2 *}$
}

${ }^{I}$ CeSI, Aging Research Center, “G. D'Annunzio'” University Foundation, Chieti, Italy; ${ }^{2}$ Molecular Biotechnology Centre, Department of Clinical and Biological Sciences, University of Turin, Turin, Italy.

*Address correspondence to: Prof. Federica Cavallo, Molecular Biotechnology Centre, University of Turin via Nizza 52, 10126 Turin, Italy; Tel 0039-0116706457; E-mail: federica.cavallo@unito.it

\begin{abstract}
Tumor-transplanted rodents are primarily adopted to study cancer progression in vivo and the inhibitory potential of the immune system. Genetically engineered, cancer-prone mice, however, more closely mimic several features of human cancer. Inbred BALB/c female mice transgenic for the rat neu (Her-2/neu, ErbB-2) oncogene (BALB-neuT mice) constitute an intensively studied mammary cancer model. Key features of this model include that (1) each of their ten mammary glands develops an independent carcinoma that slowly progresses from microscopic lesions to invasive tumors; (2) multiple in situ carcinomas are accompanied by greater dissemination of neoplastic cells into the bone marrow; (3) lung metastases are evident in the later stages; (4) over-expression of the protein product of the transgenic neu oncogene in the newborn thymus induces the deletion of $\mathrm{T}$ cell clones reacting with high-affinity against it, while the step-wise progression of mammary lesions triggers negative regulation mechanisms that suppress antibody- and perforin-mediated immune surveillance mechanisms. Thus, boosts of innate immunity delay cancer progression and vaccines administered when only early microscopic lesions are present provide lifelong protection, whereas their efficacy tails off when they are administered to mice with more advanced lesions. Because this model mimics some of the most critical features of human disease, it has been successfully used to investigate a number of therapeutic agents, including the role of adaptor proteins (P130 cap), signal transducers (STAT3), phosphoinositide 3-kinase (PI3K), and oncogenic stress sensing kinases (MKK7) in neu-driven carcinogenesis, and assessment of the efficacy of brakitherapy in the control of mammary cancer.
\end{abstract}

\section{TRANSGENIC MOUSE MODELS OF CANCER.}

Experimental models are constructed to emphasize a few basic features of a complex situation. The deeper investigation of a particular set of functions they allow provides information from which the real situation can be inferred. Their responsibility is thus enhanced because their findings influence the rationale for fresh interventions. In vivo models of cancer provide information of importance for the implementation of therapies based on fresh concepts [1].

Tumor transplantation in syngeneic healthy, young rodents is a flexible way of studying a few features of neoplastic growth. Models of this kind have highlighted the central role of cytotoxic $\mathrm{T}$ lymphocytes (CTL) in tumor rejection [2]. This information was and is still of major importance since it provides the rationale for many ongoing clinical trials aimed at the activation of CTL for tumor therapy. However, these models confer no more than a snapshot that 
distorts the tumor-host relationship owing to its unrealistic time dimension. Transplanted tumors usually grow and kill their hosts in a matter of few weeks and are thus an acute model of a relationship that in a cancer patient lasts several years. The brief time scale typical of transplantable tumors does not allow the observation of a few critical features of natural tumor growth, such as the selection of clones that no longer express the antigen targeted by the immune attack nor the histocompatibility glycoproteins required for $\mathrm{T}$ cell effector functions [3]. In addition, progressive adaptation of the immune system to the tumor antigens, the various kinds of tumor-borne immunosuppression and tumor and immune influences on the microenvironment never attain any significance in these fast models. By contrast, when the time scale of tumor-host relationship is longer, the reciprocal influences of the tumor and the immune system may shape the pace of cancer progression [4]. The limited life span of rodents as compared to humans does not permit to fully overcome the inappropriate time perspective [5]. Nevertheless, when the time is not anymore of few weeks but it is expanded to significant part of a rodent's natural lifespan, anti-tumor reaction mechanisms acquire a different, and often unforeseen importance $[4,6]$. The recent availability of cancer prone genetically engineered mice (GEM) that naturally develop a tumor as a consequence of a defined gene alteration artificially inserted in their genome provides a challenging alternative to transplantable tumor models [7].

A defined genetic predestination to develop a tumor, tumor progression through defined stages, the natural occurrence of disseminated cells and metastasis, and the long-lasting interaction with the immune system are the appealing features of cancer prone GEM models [1].

The onset and progression of these tumors often mimic several features of their human equivalents. There are, however, pitfalls that may lead to distorted conclusions. A few GEM models are too artificial, since the gene alteration is foreign to the human setting. In others, the pathogenic alteration has no exact human equivalent. Because of the peculiarities of the transgene promoter, the genetic alteration of GEM mice may not follow the same developmental expression pattern as human cancer. Moreover, genetic aberrations may be overexpressed throughout a whole organ resulting in the parallel progression of several regions toward cancer. The timing of the first expression of the transgene affects the kind and intensity of the immune tolerance to the transgene protein product. When the transgene encodes a xenogeneic tumor antigen, the question whether the tolerance to it is comparable to that to autologous antigens of human tumors acquires crucial importance. Finally, a few GEM models are too cumbersome to be studied since the tumor arises only in a small percentage of mice or after an over-long latency [1].

\section{MAMMARY CANCER PRONE BALB-neuT FEMALE MICE.}

GEM models mimic distinct features of human mammary cancer $[7,6,8]$. Because of the importance of the Her2/neu (ErbB-2) oncogene in human mammary carcinogenesis [9], and mostly thanks to the work of Leder and Muller, several lines of cancer-prone GEM overexpress the proto and the activated rat neu (r-neuT) oncogene in their mammary glands [10]. Those overexpressing the r-neu protooncogene closely reproduce the gene lesions that cause human mammary carcinoma in women, whereas those transgenic for the r-neuT have earlier tumors that grow aggressively, and thus better mimic the devastating progression, metastatizing ability, and lethality of human Her-2 mammary carcinoma [11].

Of those expressing the $r$-neuT oncogene, inbred $\mathrm{BALB} / \mathrm{c}$ mice expressing it under the transcriptional control of the mouse mammary tumor virus promoter long terminal repeats (MMTV-LTR promoter) (BALB-neuT mice) have been exploited in many experimental studies because of the consistency of the progression of their mammary lesions [1]. These 
mice were generated by mating random-bred male mouse transgenic for Muller's plasmid coding for the r-neuT oncogene [12] with a wildtype BALB/c female. Back-intercrossing of their $\mathrm{r}$-neuT ${ }^{+}$male offspring for more than 50 generations with wild-type BALB/c CR females [13] has provided the inbred BALB-neuT strain with a $100 \%$ mammary carcinoma penetrance. All females develop a mammary hyperplasia within the first month of life, and independent and progressively growing carcinomas are palpable in all their ten mammary glands within 7 months [13, 14]. The r-neuT oncogene is mostly expressed in the mammary gland, and to a much lesser extent, and not always, in the salivary and Harderian glands [12, 15]. Mammary carcinogenesis outpaces that of the salivary glands in female BALB-neuT mice, whereas metastatizing salivary tumors are the prominent cause of death in the males $[15,16]$.

\section{NATURAL HISTORY OF MAMMARY} CANCER IN BALB-neUT FEMALE MICE.

Pathological features. From the $4^{\text {th }}$ week of life, cells in the not yet completely formed ducts of the mammary glands express the protein product of r-neuT oncogene, the r-p185 ${ }^{\text {neu }}$ protein. These r-p185 ${ }^{\text {neu+ }}$ cells proliferate and form side buds along most of the ducts of the mammary gland. Expanding side buds coalesce to give rise to atypical hyperplasia and then carcinoma in situ. These lesions grow to become invasive and palpable through the skin around the $19^{\text {th }}$ week of life, and occupy the bulk of the subcutaneous fibroadipose tissue from the armpits down to the

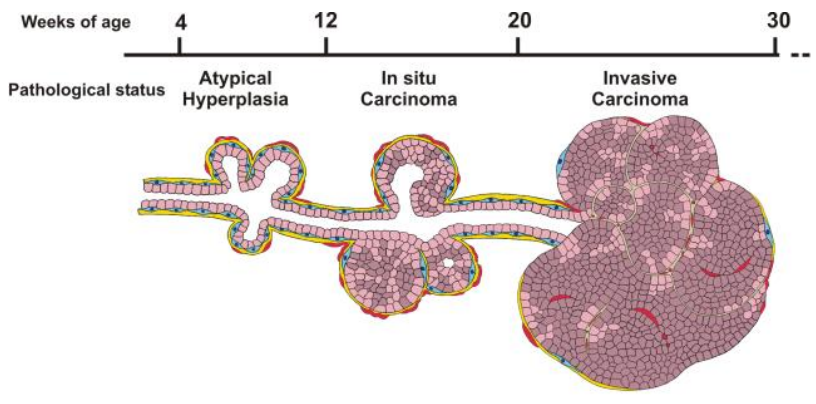

Fig. 1. Time course of mammary tumor development in BALB-neuT female mice. sites of the two sets of five mammary glands on each side of the groin (Fig. 1).

Lung metastases are evident from the $30^{\text {th }}$ week onwards. Whole mount, histological and immunohistochemical analyses are used to monitor tumor development [1].

Whole mount analyses from the $4^{\text {th }}$ to the $10^{\text {th }}$ week reveal the appearance of black spots with a roundish shape along the path of and within the ducts (Fig. 2A) that subsequently grow to form nodules very similar to the lesions of human carcinoma in situ (Fig. 2B) whose further expansion and clumping gives to masses that correspond to invasive carcinomas sometimes capable of occupying the whole of the gland and its subcutaneous tissue (Fig. 2C).

Histological examination shows that from the $4^{\text {th }}$ to the $10^{\text {th }}$ week, the small roundish spots displayed by the whole mount are side buds formed of neoplastic cells.

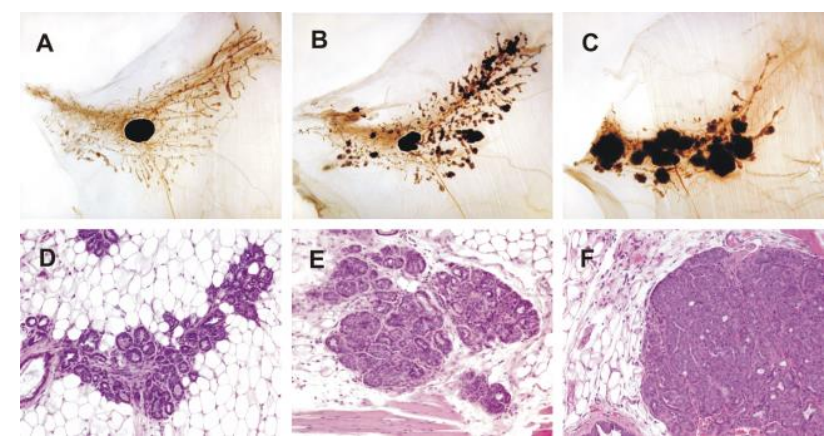

Fig. 2. Whole mount preparations (A, B, C) and hematoxylin and eosin staining $(\mathrm{D}, \mathrm{E}, \mathrm{F})$ of histological sections from BALB-neuT mammary glands at 10, 18 and 22 weeks of age. (Whole mount magnification x63, H\&E x200). At 10 weeks of age, the whole mount preparations reveal small side buds along the mammary ducts (A). At 18 weeks, these have grown and coalesced to form dark nodules (B). At 22 weeks, they are very voluminous and occupy most of the mammary fat pad (C). Histological picture at 10 weeks showing side buds composed of ductal extraflexions lined with several, occasionally atypical epithelial layers, and still surrounded by myoepithelial cells and a basement membrane (D). At 18 weeks, these buds have partly coalesced. They are larger and completely filled with neoplastic epithelial cells (carcinoma in situ) (E). At 22 weeks, they are even larger and formed of solid epithelial aggregates with a delicate interposed stroma. The masses are invading the surrounding fibroadipose tissue and are in contact with vessels and nerves $(\mathrm{F})$. 
Their basement membrane is still visible under a layer of myoepithelial cells (Fig. 2D). Around the $13^{\text {th }}-14^{\text {th }}$ week, some ducts, whose basement membrane is still distinguishable, are almost completely obliterated by neoplastic cells (Fig. $2 \mathrm{E})$ and the myoepithelial cells eventually disappear. From the $20^{\text {th }}$ week, the tumor begins to invade the surrounding fibroadipose tissues in the form of increasingly larger masses formed of clumps of cells separated by a delicate stromal tissue (Fig. 2F).

Immunohistochemical analysis discloses overexpression of $\mathrm{r}-\mathrm{p} 185^{\text {neu }}$ from the $4^{\text {th }}$ week onwards by the neoplastic cells in all the lesions and the metastases (Fig. 3A, B, C). An assay with specific anti-PCNA antibodies shows that several tumor cells are proliferating (Fig. 3D, E, F).

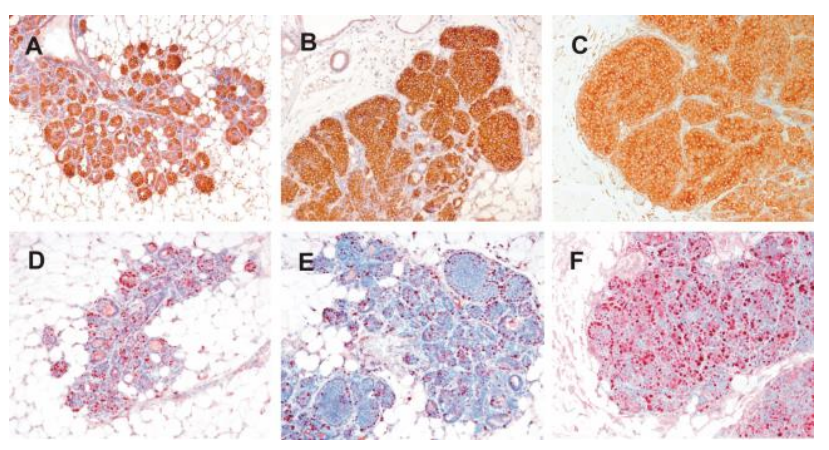

Fig. 3.Immunohistochemical staining of r-p185 ${ }^{\text {neu }}(A, B, C)$ and PCNA (Proliferative Cell Nuclear Antigen) (D, E, F) on sections from BALB-neuT mammary glands at 10, 18 and 22 weeks of age (x 200). Immunohistochemical staining with anti r-p185 ${ }^{\text {neu }}$ monoclonal antibody shows constant r-p185 $185^{\text {neu }}$ overexpression by the neoplastic cells. Anti-PCNA staining reveals proliferative activity in numerous neoplastic cells.

The combined histological and immunohistochemical picture shows that the number of stem cells $\left(\mathrm{Scal}^{+}, \mathrm{Musashi}^{+}\right)$is greater in the atypical hyperplasia and carcinoma in situ stages as opposed to the invasive carcinoma stage. The neoplastic cell proliferation index is very high in the early stages, especially during atypical hyperplasia, and the bordering stroma is copiously vascularized, whereas in the advanced stages a high index is only found in the more peripheral areas in contact with the stroma and on the tumor expansion front.

The atypically hyperplastic ductules (weeks 410 of age) are surrounded by many macrophages $\left(\mathrm{CD} 11 \mathrm{~b}^{+}, \mathrm{CD} 8^{+/}\right)$, mainly in close contact with the numerous small vessels running in the delicate branches of the stroma. A few T CD4 ${ }^{+}$ lymphocytes are also present. They remain confined to the outer borders of the growing in situ and invasive carcinomas, whereas numerous macrophages persist in the stroma.

$\mathrm{r}-\mathrm{p} 185^{\text {neu+ }}$ neoplastic cells begin to disseminate from the primary tumor during the $4^{\text {th }}$ to $9^{\text {th }}$ weeks of life, when it is in the atypical hyperplasia stage. They pass beyond the basement membrane, invade the stroma (Fig. 4), and enter the blood and lymph vessels. In addition, r-p185 ${ }^{\text {neu }}$ cells spread to the bone marrow. As already stated, therefore, dissemination begins in the initial stages of carcinoma growth when its intensity is greater than when the tumor is bigger.

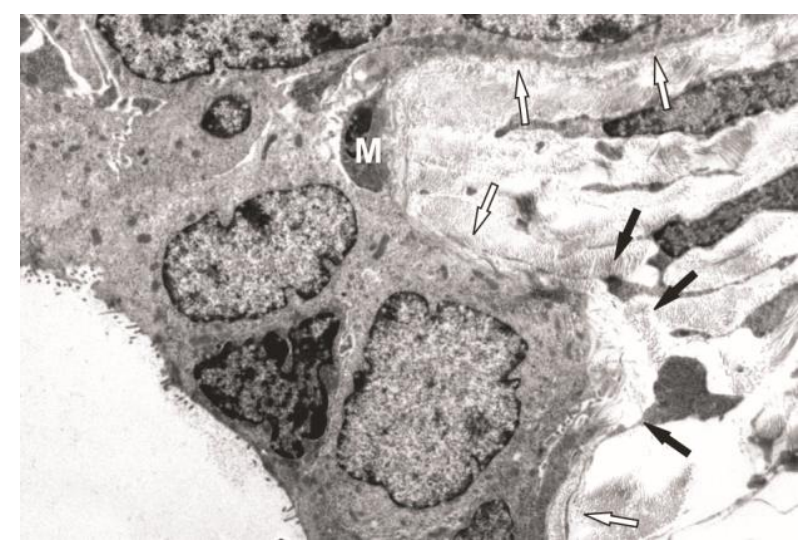

Fig. 4. Electron microscopy of a side bud from a 9-week-old BALB-neuT female mouse. A dysplastic luminal cell with its basal portion is passing through the interrupted basement membrane (black arrows), which is in direct contact with fundamental substance and collagen fibrils of the stroma. Above there is a myoepithelial cell (M) between the membrane (white arrows) and the ductal epithelial cells.

Genomic hybridization has demonstrated that $\mathrm{r}$ p185 ${ }^{\text {neu }}$ cells disseminated in the bone marrow are rendered extremely dissimilar by their display of a variety of chromosome aberrations [17]. These aberrations, and those in the cells of the lung metastases, are not the same as those of the primary from which they must certainly be 
derived. Metastases, in other words, stem from genomically and chromosomally dissimilar cells that acquire further genetic alterations, partly influenced by their microenvironment, as their proliferation proceeds. The lungs are thus preferentially colonized by cells whose characteristics differ from those of the cells that invade the marrow.

The observation of $\mathrm{r}-\mathrm{p} 185^{\text {neu+ }}$ cells in the marrow is not an unequivocal indicator of the presence of frank metastases, nor is it yet clear whether they subsequently form lung metastases, though these are more likely built up by waves of r-p $185^{\text {neu+ }}$ cells brought in by the blood. In BALB-neuT females, in fact, clinically evident metastases are only found in the lungs. Here, neoplastic cells initially settle in capillaries and vessels before proliferating to form roundish intravascular nodules (Fig. 5B) that eventually invade the parenchyma (Fig. 5C).

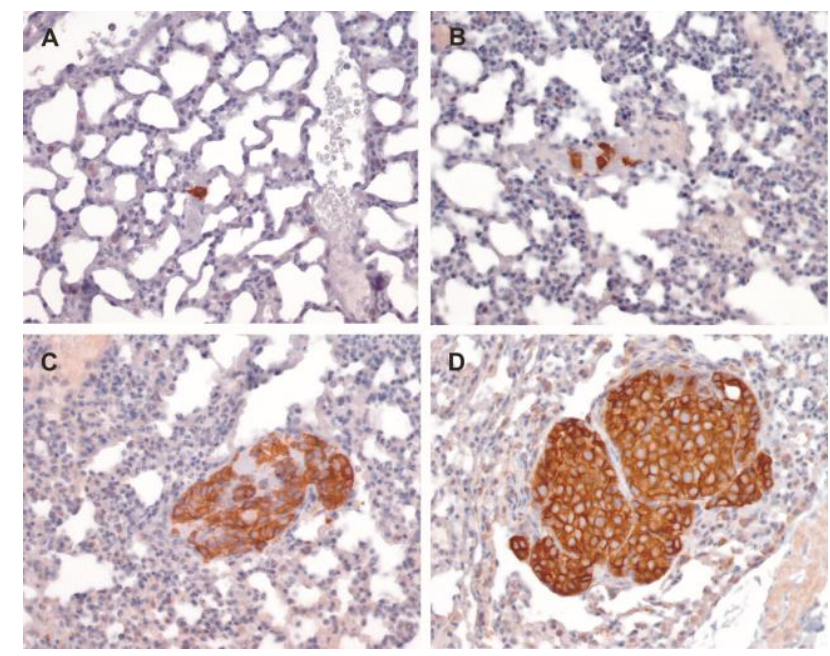

Fig. 5. Immunohistochemical staining of r-p185 neu during metastatization of the lung by mammary neoplastic cells: (A) r-p185 $5^{\text {neut }}$ cell in a dilated lung capillary full of fibrin and activated endothelia; (B) dilated lung capillary with a string of three $\mathrm{r}-\mathrm{p} 185^{\text {neu+ }}$ cells presumably brought in by successive waves of neoplastic dissemination; (C) proliferation of $r$ $\mathrm{p} 185^{\text {neu+ }}$ cells to form solid aggregates with fibrin still interposed; (D) metastatic proliferation is mostly intravascular, with subsequent invasion of the parenchyma.

Comparison with human breast tumors: differences and similarities. There are both morphological differences and similarities between this murine model and mammary carcinoma in women. This, in fact, usually appears in only one breast and in a single site, most frequently the external superior quadrant, whereas in BALB-neuT mice there is widespread proliferation along all the ducts of every breast, and the larger tumor masses are partly the result of coalescence of small nodules located in various portions of the ducts. As in the human tumor, on the other hand, the neoplastic cells are rendered dissimilar through their ability to express different molecules in function of the genic alteration they have acquired, and also through their crosstalk with the tumor microenvironment.

All the neoplastic cells in the BALB-neuT model, however, overexpress $r-p 185^{\text {neu }}$, whereas a substantial percentage of those in what are still classed as Her $2^{+}$human mammary tumor are actually Her2- (Fig. 6). Moreover, the over expression of r-neuT in the BALB-neuT model seems the only cause of the neoplastic transformation, whereas the human carcinogenesis is apparently due to accumulation of several genetic alterations.

Neoplastic dissemination in the human bloodstream may be an early finding, and neoplastic cells are detected in the bone marrow of many breast tumor patients. This detection is indicative of a poor prognosis, whereas the presence of such cells in the blood has not yet been established as a sure sign of tumor progression.

The BALB-neuT model permits investigation of both dissemination and metastatization, though the latter is confined to the lungs in the mouse, whereas many other human organs (bones, liver, adrenals, brain and meninges) are colonized.

Dissemination, too, is a constant feature of the murine atypical hyperplasia and carcinoma in situ stages, whereas in humans it is probably less frequent, even if disseminated cells are found in the blood and bone marrow of some patients with very small and indeed still in situ tumors.

\section{IMMUNE MODULATION IN BALB- neuT MICE.}

The expression of transgenic r-neuT is the only genetic difference between BALB-neuT and 
wild-type BALB/c mice.

Their immune systems can thus be directly compared to assess the consequences of both expression of $\mathrm{r}-\mathrm{p} 185^{\text {neu }}$ and the progression of $\mathrm{r}-$ p185neu ${ }^{+}$lesions.
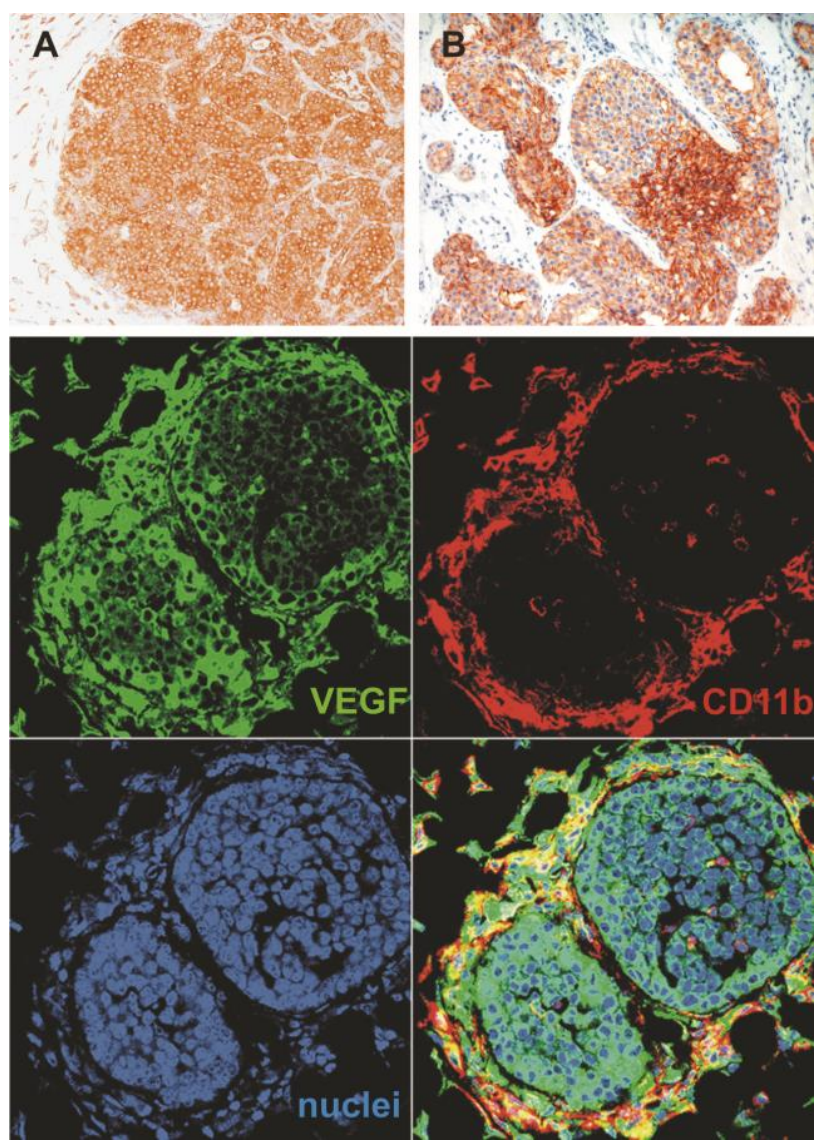

Fig. 6. Immunohistochemical staining of $\mathrm{p} 185^{\text {neu+ }}$ in mammary tumors from a BALB-neuT female mouse (A) and a human patient (B), and of VEGF and CD11b ${ }^{+}$on an 18week BALB-neuT neoplastic lesion. p185 ${ }^{\text {neu }}$ is uniformly expressed in an all the neoplastic murine epithelial cells (A), whereas in the human setting positive and negative cells coexist even in cases classed as highly c-ErbB-2 positive $\left(\right.$ ErbB-2 $\left.{ }^{+++}\right)$(B). Despite their uniform r-p185 ${ }^{\text {neu }}$ expression, the murine tumor cells display markedly dissimilar production of angiogenic factors such as VEGF. Confocal microscopy shows that VEGF (green) is produced by macrophages $\left(\mathrm{CD}_{11 b^{+}}\right.$cells in red), and only by the neoplastic cells closest to the stroma and the macrophages themselves.

Central tolerance to r-p185. The first critical change due to this expression is acquisition of a central tolerance to $\mathrm{r}-\mathrm{p} 185^{\text {neu }}$. In newborn BALB-neuT mice, transgenic $\mathrm{r}-\mathrm{p} 185^{\text {neu }}$ is markedly and diffusely overexpressed by the thymic epithelium, whereas in adult mice it is mostly overexpressed mostly by mammary and salivary tissues. This has a dramatic impact on the repertoire of $T$ cells that reach the periphery: $\mathrm{CD}^{+} \mathrm{T}$ cell clones that in wild-type BALB/c mice interact at high avidity with the dominant $\mathrm{H}-2 \mathrm{~K}^{\mathrm{d}} \mathrm{r}-\mathrm{p} 185^{\text {neu }}$ 63-71 epitope are missing in BALB-neuT mice. Immunization against $r$ p185 neu elicits high affinity $\mathrm{CD} 8^{+} \mathrm{CTL}$ in wildtype $\mathrm{BALB} / \mathrm{c}$ mice compared with only a marginal CTL response in BALB-neuT, whose immune response elicited by vaccines rests almost solely on activation of $\mathrm{CD}^{+} \mathrm{T}$ cells. Differences in thymic selection appear to be responsible for this split tolerance leading to selective deletion of $\mathrm{CD}^{+} \mathrm{CTL}[18,19]$.

Expansion of immature myeloid cells. The lengthy stepwise progression of mammary lesions in female BALB-neuT mice is accompanied by expansion of $\mathrm{Gr}^{+} 1^{+}$(Ly6G), $\mathrm{Mac}^{+} 1^{+}$(CD11b), ER-MP12+ (CD31) immature myeloid cells in the peripheral blood and spleen, whose number increases marginally during the pre-neoplastic stages, then markedly when invasive carcinomas become palpable in the mammary pad and progress to large neoplastic masses. This relatively late expansion rests on the enhanced release by carcinoma cells of vascular endothelial growth factor and other factors, while its progressive inhibition of T cells results in their failure to respond to alloantigens and CD3 triggering [20].

Expansion of $T$ regulatory (Treg) cells. The progression of r-neuT-driven carcinogenesis in the mammary glands of BALB-neuT female mice is also accompanied by a progressive expansion of $\mathrm{CD}^{+}, \mathrm{CD}^{2} 5^{+}, \mathrm{Foxp}^{+}, \mathrm{GITR}^{+}$Treg cells. Treg expansion is not evident or marginal until the appearance of hyperplasia and carcinomas in situ, but is rapid in both the spleen and the mammary lesions when these coalesce and progress to invasive carcinomas. This expansion can be seen as a natural response designed to physiologically dampen the autoimmune reaction elicited by anomalous 
overexpression of $\mathrm{r}-\mathrm{p} 185^{\text {neu }}$ by the expanding mammary lesions [21].

Perforin-dependent, $N K$ and T cell surveillance. Despite the extreme oncogenicity of this model and the profound central tolerance to r-p185 ${ }^{\text {neu }}$, immune surveillance mechanisms affect the onset of mammary carcinomas in BALB-neuT mice. Mammary lesions developing in BALBneuT mice as such and those in which the perforin gene has been knocked out (BALBneuT/pfp KO) display the same level of $r$ p185 ${ }^{\text {neu }}$ expression. In the latter, however, carcinogenesis is accelerated. The latency time of the first mammary tumor is shorter, the progression from hyperplasia to invasive neoplasia is quicker, and the number of tumors per mouse is greater than in BALB-neuT mice. Both NK cells and $\mathrm{CD}^{+} \mathrm{T}$ cells are major populations that express the perforin effector molecule. Depletion of NK, but not $\mathrm{CD}^{+}$or $\mathrm{CD}^{+}$cells, speeds carcinoma onset $[22,23]$ suggesting that NK cells are the key perforinexpressing effector cells that delay mammary carcinoma development in this model [22]. However, other experiments have revealed accelerated carcinoma progression in mice depleted of both $\mathrm{CD}^{+}$and $\mathrm{CD}^{+}{ }^{+} \mathrm{T}$ cells [23].

The decline of immune surveillance. These data do much to indicate that cell-mediated immune surveillance impairs, but fails to fully prevent mammary carcinogenesis in these mice. Expression of the r-neuT oncogene into every mammary gland leads to continuous generation of tumor cells that should be kept at bay. This endless struggle between a tumor and an immune system eventually results in the expansion of regulatory mechanisms whose onset tips the scales against natural responses.

IL-13 mediates the negative regulatory effect of type II, Va14- TCR negative NKT cells [24]. When BALB-neuT female mice are treated with a soluble IL-13 inhibitor (sIL-13Ra2-Fc) from the stage of diffuse atypical hyperplasia until an invasive tumor is palpable, this IL-13 blockade improves immune surveillance and retards the appearance of mammary carcinomas. Even if the origin of IL-13 and its mechanisms of action were not yet defined in the BALB-neuT model, it was previously observed that IL13 released by type II NKT cells naturally primed by tumor growth may contribute to the suppression of immunosurveillance by inducing myeloid cells to enhance their suppressive activity by releasing TGF- $\beta$. The marginal expansion of immature myeloid cells during the early stages of mammary lesions may be enough to amplify the effects of this negative regulatory circuit [23].

The weight of regulatory circuits in the abrogation of natural immunosurveillance becomes even more evident following chronic Treg depletion started in these early stages [21]. BALB-neuT mice bearing mammary lesions of increasing size display marginal if any production of antibody and $\mathrm{CD}^{+} \mathrm{T}$ cell mediated citotoxicity against r-p185 ${ }^{\text {neu }}$. However, when they receive repeated administrations of anti-CD25 monoclonal antibodies and undergo chronic depletion of Treg cells, a significant antibody and CTL mediated natural immunosurveillance against $\mathrm{r}$ p185 ${ }^{\text {neut }}$ tumors is unveiled. When r-neuT carcinogenesis takes place in the absence of Treg cell expansion, it is delayed by an unhampered natural response [21].

This presence of a natural antibody and $\mathrm{CD}^{+} \mathrm{T}$ cell mediated surveillance is apparently in conflict with the evidence of a central tolerance to $\mathrm{r}-\mathrm{p} 185^{\text {neu }}$ acquired by BALB-neuT mice. The natural CTL response that follows Treg removal is due to low-avidity $\mathrm{CD} 8^{+} \mathrm{CTL}$ expressing an otherwise cryptic $\mathrm{T}$ cell repertoire. However, expansion of these CTL that have escaped thymic selection is normally concealed by Treg cell expansion in the periphery. Treg depletion allows them to expand and display a CTL response. Treg depletion also spurs spontaneous expansion of $\mathrm{CD}^{+} \mathrm{T}$ cells specific for $\mathrm{r}-\mathrm{p} 185^{\text {neu }}$ [19].

Cytokine modulation of r-neuT carcinogenesis. Penetrance of r-neuT provides a major oncogenic signal. The features of mammary tumor progression, however, are markedly 
modulated by cytokines in the tumor microenvironment. Microarray data show that a transcriptional signature associated with proinflammatory molecules can be defined during r-neuT-driven carcinogenesis [25]. When this signature is applied to a collection of human breast cancer data [26], it arranges them in function of different types or degrees of leukocyte infiltration.

Proinflammatory activation in the mammary glands of BALB-neuT mice may thus reflect a general pattern of human breast cancer. Ingenuity analysis of genes associated with the tumor microenvironment identifies interferon (IFN) $-\gamma$ as one of the four hub cytokine genes whose increased expression in this microenvironment is naturally associated with mammary cancer progression.

These findings suggest that IFN- $\gamma$ acts as an additional natural surveillance mechanism that impairs r-neuT carcinogenesis by restraining tumor-driven neoangiogenesis [25]. In IFN- $\gamma$ KO BALB-neuT mice, in fact, progression is quicker and numerous, larger-than-normal caliber vessels covered with pericytes are present both inside and around the tumors. Tumor necrosis factor (TNF) is another hub cytokine gene whose expression increases during BALB-neuT mammary carcinogenesis [25].

While IFN- $\gamma$ naturally inhibits r-neuT carcinogenesis, TNF plays a critical role in tumor promotion as shown by various mouse models $[27,28]$. When BALB x C57BL/6 F1 rneuT mice are reconstituted with bone marrow cells from C57BL/6 mice KO for the TNF gene, $\mathrm{r}$-neuT carcinogenesis is significantly impaired. In the absence of leukocyte-derived TNF, intratumoral vessels are disarranged, lack pericyte coverage and induce diffuse vascular hemorrhage and stromal necrosis [28].

\section{EXAMPLES OF THE USE OF BALB- nEUT MICE IN TUMOR IMMUNOLOGY.}

The aggressiveness and consistency of r-neuTdriven mammary carcinogenesis make BALBneuT female mice one of the models most frequently used to study immunity to autochthonous mammary cancer. The data outlined above show that overexpresson of the $r$ neuT oncogene and the development of mammary carcinomas trigger multiple immune regulatory activities that affect the pace of cancer progression. As the tumor expands, suppressive regulatory circuits obscure the significance of cellular and antibody-mediated surveillance. Moreover, a delicate balance between the cytokines in the tumor microenvironment decides the pace of carcinogenesis, primarily by restraining or favoring tumor neoangiogenesis.

The passage from each stage of the tumorimmune system relationship to the next recapitulates several aspects of the dynamics of immune modulation in cancer patients. A cell and antibody-mediated reaction to tumoroverexpressed Her-2 is evident in women with mammary cancer [29] while rampant immunesuppression is a common feature of patients with advanced tumors [30].

The interactions with immune mechanisms that shape the progression of mammary cancer make BALB-neuT mice an appealing model for assessment of the potential and limits of immune interventions designed to modify the tumor microenvironment, boost of host innate immunity, and find ways of overcoming immune tolerance to tumor associated antigens.

Systemic IL-12. Modulation of the innate immunity of BALB-neuT mice by prolonged administration of IL-12 (5 daily intraperitoneal injections of $100 \mathrm{ng}$, one week on, 3 weeks off) significantly delays mammary carcinogenesis and reduces tumor multiplicity mainly by inhibiting tumor-induced angiogenesis [11]. This antiangiogenic effect is stronger on the fragile capillaries that accompany the shift from the preneoplastic to the neoplastic condition, whereas the mature and differentiated vessels of the more advanced neoplastic lesions are less susceptible [31]. It is primarily the result of IL12 's ability to induce high and sustained serum levels of IFN- $\gamma$, a goal that can be reached by administering low doses of IL-12 [32]. 
Local and systemic CpG. Modulation of the tumor microenvironment with Toll-like receptor (TLR) ligands such as non-methylated-CpG oligodeoxynucleotides (CpG-ODN) induces a strong local reaction that persistently impair mammary carcinogenesis. Starting when in situ carcinomas were scattered over all the mammary glands of BALB-neuT mice, CpG-ODN 1826 were injected in the stroma of the fourth left mammary gland six times at four week intervals. At one year of age, $67 \%$ of these glands were tumor-free, and an impressive reactive infiltrate of macrophages, $\mathrm{CD}^{+}, \mathrm{CD}^{+}$, and $\mathrm{B} 220^{+}$cells formed a carpet around the precancerous lesions. This treatment both delayed the onset of carcinomas in the injected glands, and also extended the latency of tumors in glands both close and far from the injection site [33]. In effect, a major drawback on the use of $\mathrm{CpG}$ is that intratumoral injection is not always possible. To target GpG-ODN to a tumor site anywhere within the body a hybrid between a monoclonal antibody directed against $\mathrm{r}-\mathrm{p} 185^{\text {neu }}$ (7.16.4 from D. M. Greene) [34] and CpG-ODN has been produced. Its intravenous injection leads mice to rejection of a challenge of syngeneic r-p185 ${ }^{\text {neu+ }}$ tumors on the part of $30 \%$ of BALB-neuT mice. Combination of GpGODN with the removal of Treg cells leads all BALB-neuT mice to reject the tumor challenge and acquire a tumor-specific immune response [35].

Zoledronic acid (ZA). ZA is the most potent nitrogen-containing bisphosphonate. Lifelong intravenous administration of clinically compatible doses of ZA to BALB-neuT mice once a week for 4 consecutive weeks, repeated after 3 weeks of rest, induces a significant increase in tumor-free and overall survival by modifying the tumor microenvironment. The tumor stroma of ZA-treated mice did not show significant differences in the number of infiltrating $\mathrm{CD}^{+}$and $\mathrm{CD}^{+} \mathrm{T}$ lymphocytes, $\mathrm{B}$ lymphocytes, Treg cells, granulocytes and gamma-delta $\mathrm{T}$ cells. By contrast, $\mathrm{CD} 11 \mathrm{~b}^{+}$ macrophages associated with the mammary lesions were strongly reduced and displayed a dramatically reverted polarization from M2 to M1 phenotype. They no longer produce IL-10 and become strongly positive for IFN $-\gamma$. This repolarization of macrophages greatly influences the pace of r-neuT carcinogenesis since it is associated with both a reduced production of VEGF and a markedly restrained neovascularization of mammary lesions [36].

Oral human lactoferrin. Lactoferrin is an ironbinding glycoprotein present in milk, tears, nasal exudates, saliva, bronchial mucus, gastrointestinal fluids, cervicovaginal mucus and seminal fluid. It is also secreted by neutrophils and present at high levels at sites of bacterial infection. The recombinant human lactoferrin produced in Aspergillusniger (Talactoferrin, TLF) differs from native human lactoferrin only in the nature of its glycosylation. When BALB-neuT mice received TLF (3 $\mathrm{mg} / \mathrm{m}^{2}$ ) administered orally by gavage once a day, 5 days per week, 3 weeks on and 1 week rest until mammary tumors were evident, their progression was markedly delayed. The diseasefree survival was extended and tumor multiplicity was reduced [37].

Anti r-p185 $5^{\text {neu }}$ vaccines. The consistent stepwise progression of mammary cancer in BALB-neuT mice provides a convenient model with which to weigh the preventive and therapeutic efficacy of anti Her-2 vaccines. Straightforward assessment of the potential and limits of the immunity induced is obtained by immunizing mice with mammary lesions of progressive size.

a. DNA vaccines. Injection of DNA plasmids coding for portions of r-p185 ${ }^{\text {neu }}$ [38], their combination with adjuvants of different kinds [39, 40, 41], and the injection of plasmids encoding fusion proteins consisting of a portion of human $\mathrm{p} 185^{\text {neu }}$ fused to the extracellular domain of CTLA-4 to better target their delivery to antigen-presenting cells [42] elicit a significant protection against the onset of mammary lesions. Stronger protection is obtained when injection of plasmids encoding for the extracellular and transmembrane 
domains of $\mathrm{r}-\mathrm{p} 185^{\text {neu }}$ is followed by intramuscular electroporation [43, 44]. Their combination with weekly administration of IL12 enables vaccination to provide protection at late points in time when vaccine electroporation alone is ineffective [45]. Through electroporation of plasmids encoding progressively smaller portions of $r-p 185^{\text {neu }}$ the smaller epitopes still able to elicit a marked protection are defined [46]. Electroporation at ten-week intervals of plasmids coding for a chimeric rat-human $\mathrm{p} 185^{\text {neu }}$ molecule provides lifelong protection in more than $50 \%$ of BALBneuT mice [47]. The protection afforded by plasmid electroporation is enhanced by its combination with CpG-ODN SM360320, an agonist of TLR 7 [48] or a new CpG-ODN antagonist of TLR 9 [49].

b. Cell vaccines. A significant and persistent protection is also afforded by cellular $[50,51]$ and DNA [52] vaccines combining r-p185 ${ }^{\text {neu }}$, an alloantigen, with IL-12. A strong protection is also elicited by vaccination with dendritic cells engineered to express a portion of r-p185 ${ }^{\text {neu }}$ [53]. Comparison of the protection afforded by vaccination with murine NIH3T3 cells engineered to express rat or human $\mathrm{p} 185^{\mathrm{neu}}$, or human EGFR, ErbB-3 and ErbB-4 showed that the best protection was induced by cells expressing $r-p 185^{\text {neu }}$. Lower protection was elicited by NIH3T3 expressing human $p 185^{\text {neu }}$ and the three ErbB receptors [54].

c. Peptides. Immunization with conformational peptide constructs that mimic regions of the dimerization loop of the $\mathrm{r}-\mathrm{p} 185^{\text {neu }}$ constructs (peptide 266-296; 298-333; 315-333) elicits high-affinity antibodies that inhibit $\mathrm{r}-\mathrm{p} 185^{\text {neu }}$ dimerization and its signaling pathways, mediate antibody-dependent cellular cytotoxicity and reduce carcinoma onset [55]. Another conformational peptide (597-626) constructed to include the native disulfide bonds to more closely mimic the native structure of $\mathrm{r}-\mathrm{p} 185^{\text {neu }}$ inhibits carcinoma onset more efficiently than its linear counterpart [56]. Significant preventive immunity is also elicited by repeated vaccinations with a synthetic peptide of $r$ p185 ${ }^{\text {neu }}$ in combination with the Toll-like receptor antagonist CpG-ODN1826. CTL response and antitumor effects become evident when the function of Treg cells is blocked [57]. d. Viruses. Virus-like particles containing extracellular and transmembrane domain of human $r-p 185^{\text {neu }}$ [58], recombinant vaccinia virus encoding full-length human $\mathrm{p} 185^{\text {neu }}$ [59], or vaccination with adenovirus encoding wild-type $[60,61]$ or codon usage-optimized [62] portions of $\mathrm{r}-\mathrm{p} 185^{\text {neu }}$ are other convenient ways of breaking tolerance and inducing a persistent immune response.

The protection afforded by these vaccines usually stems from the induction of anti-r$\mathrm{p} 185^{\text {neu }}$ antibodies that inhibit tumor progression by blocking the signals delivered by $\mathrm{r}-\mathrm{p} 185^{\text {neu }}$ $[55,56,60,63-65]$ or recruiting antibodydependent cellular toxicities [44, 49, 55, 56]. They occasionally elicit a CTL response probably due to their ability to trigger the lowavidity $\mathrm{CD}^{+} \mathrm{T}$ cells that escape central tolerance $[49,57,58,61]$. This rare response is markedly enhanced by concomitant removal of Treg cells $[19,35,57]$.

Whole transcriptome characterization of the effect of anti-r-p185 ${ }^{\text {neu }}$ vaccines. The first whole transcriptome characterization of BALB-neuT mammary cancer was published in 2004 [63] It showed that a combined DNA and cell vaccine persistently inhibited breast cancer lesions. The further observation of a strong concordance between whole-mount morphologic findings and gene expression patterns indicated that the reaction elicited by anti $r-p 185^{\text {neu }}$ vaccines elicit a reaction halts the progression of preneoplastic lesions, coupled with the reversion of mammary lesions to the stage when vaccination began (see below). Attention was also drawn to the presence of a group of genes generically pertinent to the humoral response and encoding antibody-related genes only in the vaccinated mice. This paper was soon followed by another [66] that reported prevention of multifocal mammary carcinogenesis by means of a vaccine combining IL-12 and allogeneic p185 ${ }^{\text {neu+ }}$ mammary carcinoma cells. It was also found that transcription profiling of mammary tissue from 
vaccinated mice displayed a gene expression signature different from that of untreated, tumorbearing mice, but similar to that of normal/hyperplastic mammary glands. The tumors which develop in the r-neuT mice have a basal-like signature, in agreement with human data. The BALB-neuT model thus also mimics human Her-2-induced tumorigenesis in its similarity to basal-like tumors. Subsequent work [67] showed that combination of the two microarray sets derived from the two immunization protocols applied in two laboratories to the same breast cancer model revealed their clear correlations between the increase of tumor mass and the transcription profiling. It was also clear that both protocols induced a strong, polyclonal antibody response and halted tumor growth at a condition very similar to that at which the vaccination began. Differences in the expression profiles were mainly related to the levels of a few chemokines and T-cell-specific genes that may be in some way correlated with the efficacy of the vaccination. Furthermore, combination of the expression data with the protection results indicated that chronic vaccination is needed to maintain an active IFN $\gamma$-mediated response in the mammary gland.

An anti-angiomotin vaccine. Electroporation of BALB-neuT mice with a DNA vaccine targeting the angiostatin receptor angiomotin (Amot) expressed in tumor and placental endothelium breaks tolerance and elicts antibodies that detect Amot on the surface of endothelial cells. While the immune response elicited has no significant effect on the progression of mammary carcinogenesis progression, significant alterations were evident in the morphology of the vessels associated with it. Combination of anti $\mathrm{r}-\mathrm{p} 185^{\text {neu }}$ and anti-Amot vaccination provides stronger and more persistent prevention, More than $80 \%$ of BALB-neuT mice remained cancer-free for over 70 weeks [68].

Identification of new oncoantigens. Microarray data extensively monitor gene expression in human tumors and their murine counterparts, and hence can be used to identify new tumorassociated-antigens for targeting by vaccines whose efficacy and safety can easily be evaluated in mice. A meta-analysis of the human and murine data provided the first time rough version of a procedure for identification of new tumor antigens by tagging murine tumorassociated genes in function of the correlation between their expression and the increase in tumor mass, and then selecting those with a limited expression in normal human tissues [69]. Subsequent refinement of this approach $[70,71]$ resulted in two major changes:

I) the amount of human breast cancer samples used to associate with the human disease the "tumor-associated genes" tagged in the BALBneuT model was massively enlarged;

II) The behavior of the putative TA in human pathological and normal tissue was evaluated with a reference set of genes. Specifically a wellknown set of oncoantigens, i.e. antigens with a causal role in the promotion of tumor progression [6] was used. The ErbB family was employed as the benchmark for identification of new oncoantigenes, i.e. genes characterized by an expression profile comparable to or higher than that of ErbB family members in human breast cancer, and comparable to or lower than that observed of these members in normal human tissues. This approach identified a set of interesting candidates as new oncoantigens [70]. Transcription profiling of BALB-neuT mice has thus indicated the enormous amount of knowledge that can be retrieved by combination of well addressed biological questions and a very robust cancer model.

\section{EXAMPLES OF THE USE OF BALB- neuT MICE IN ONCOLOGY}

A model acquires special significance when it is exploited by several laboratories to study distinct issues since integration of their datasets may provide a comprehensive view of the problem studied. Progressive definition of the in vivo features of tumor growth and those of the tumor-host immune relationship is the subject of studies designed to elucidate a few molecular features of Her-2 carcinogenesis. 
Role of p130Cas. p130Cas is an adaptor molecule that regulates cell motility and invasion. It is overexpressed in a significant subset of human breast cancers, while association of its high levels with Her-2 expression correlates with elevated proliferation. Transgenic mice overexpressing p130Cas in their mammary glands display extensive mammary epithelial hyperplasia during development and pregnancy, and delayed involution at the end of lactation. When they are crossed with BALB-neuT mice, doubletransgenic mice develop mammary tumors with a shorter latency than their wild-type $\mathrm{r}$-neuT $\mathrm{T}^{+}$ littermates. Western blot analysis of from primary cultures of cells from the tumors of double transgenic mice showed that p130Cas is highly overexpressed. Moreover, they displayed increased levels of tyrosine-phosphorylated proteins as well as active Src, whereas Erk1/2 MAPK activities were unaffected. Phosphorylation of PTEN, Akt, and Gsk3-h was also much more induced [72]. To further prove the weight of p130Cas in the growth of r-neuTdependent spontaneous tumors, 12-week-old BALB-neuT mice displaying many mammary in situ carcinomas received $50 \mu \mathrm{l}$ of p130Casstabilized siRNAs in their 4th-gland ductules by intranipple injection. Whole-mount analysis 6 weeks later showed that these glands developed significantly less severe lesions than those glands injected with control siRNAs. Western blot analysis of mammary gland extracts revealed that p130Cas was effectively knocked down and its down-regulation correlated with decreased Fak phosphorylation, Src and Akt activation, and reduced level of cyclin D1. These data indicate that p130Cas contributes to the accelerated ErbB-2-induced transformation, while its silencing during the progression of spontaneous ErbB-2 tumors impairs their growth [73].

Role of Signal transducer and activator of transcription 3 factor (STAT3). STAT3 is constitutively active in a high percentage of human tumors, including mammary adenocarcinomas, and its participation in Her-2 oncogene signaling has been reported. When BALB-neuT mice were crossed with mice in which Stat3 was selectively deleted in the mammary epithelium, the same rate of mammary carcinoma growth and progression was displayed by Stat3-sufficient and Stat3deficient mammary glands. This suggests that Stat3 is not required for r-neuT-driven mammary tumorigenesis [74]. In the opposite situation, to assess the potential cooperation of an activated Stat 3 with the r-neuT oncogene in mammary tumorigenesis, BALB-neuT mice were intercrossed with mice carrying a mutant, constitutively active $S$ tat $3^{\mathrm{C}}$ allele that replaces the endogenous wild-type allele. r-neuT Stat $3^{\mathrm{C} / \mathrm{WT}}$ mice developed tumors significantly earlier than their $\mathrm{r}$-neuT/Stat $3^{\text {WT/WT }}$ control littermates. At 12 weeks of age, rneuT/Stat $3^{\mathrm{C} / \mathrm{WT}}$ mice already presented invasive carcinomas, with tumor cells grouped in solid masses and small aggregates invading the surrounding fibroadipose tissue, whereas only atypical hyperplastic foci and few in situ carcinomas were detected in the rneuT/Stat $3^{\mathrm{WT} / \mathrm{WT}}$ mice. These earlier tumors display a proliferation rate similar to that of $r$ neuT Stat $3^{\mathrm{WT} / \mathrm{WT}}$ mice, but an appreciably reduced apoptosis.

Stat $3^{\mathrm{C}}$ fails to induce tumorigenesis on its own. It does, however, cooperate with the r-neuT oncogene by providing protection from apoptosis, a probable source of the earlier tumor onset, and promoting tumor aggressiveness and metastatic potential [75].

Role of phosphoinositide 3-kinase (PI3K). The PI3K pathway controls metabolism and cell growth. It is also involved in the Her-2-mediated proliferation of mammary gland epithelium, while mutations of the PIK3K gene are associated with ErbB-2 amplification. Class IA $\mathrm{PI} 3 \mathrm{Ks}$ are heterodimers composed of a catalytic subunit encoded by the PIK3CA, PIK3CB, and PIK3CD genes, and an Src homology 2, domain-containing adaptor protein. To study the importance of the link between PI3KCB and ErbB-2 signaling, BALB-neuT mice were 
intercrossed with mutant mice expressing a catalytically inactive PIK3CB (PIK3CB ${ }^{\text {K805R }}$ mice). While the mammary lesions of $r$ neuT/PIK $3 \mathrm{CB}^{\mathrm{K} 805 \mathrm{R}}$ mice display the same expression of $r-p 185^{\text {neu }}, r-n e u T / P I K 3 C^{K 805 R}$ mice displayed a strong reduction of the early atypical hyperplasia. At later stages, foci of transformation, the numbers of proliferating cells, the tumor multiplicity and the rate of tumor growth were still reduced. When the cells of autochthonous tumors are cultured in vitro, those from r-neuT/PIK3CB ${ }^{\mathrm{K} 805 \mathrm{R}}$ tumors grow significantly slower than controls. Taken as a whole, these data suggest that restrained growth of $\mathrm{r}$-neuT tumors is intrinsic to the function of the PIK3K gene in the mammary gland epithelium [76].

Role of MKK7 kinases. Oncogenic stress activates the DNA damage response and the key tumor suppressor p53, which prohibits tumor growth. To assess the function of stress kinase signaling in epithelial tumorigenesis Map $2 \mathrm{~K} 7$ was deleted in mammary epithelial cells. These mice (Map2K $7^{\text {Dmamm }}$ mice) were crossed with BALB-neuT mice. r-neuT/Map $2 K 7^{\text {Dmamm }}$ mice displayed a much earlier onset of mammary carcinomas. Moreover their overall survival was markedly reduced compared to control littermates. At 16 weeks of age, their mammary glands of r-neuT/Map $2 K 7^{\text {Dmamm }}$ harbored many carcinomas compared with only a few lesions in the controls. These results show that deletion of Map2K7 facilitates r-neuT driven carcinogenesis, whereas MKK7 is a potent mammary tumor suppressor [77].

Therapeutic effect of brachytherapy. Brachytherapy (from the Greek "brachys" = short) refers to the use of radionuclides within or close to a tumor: a pre-targeted tissue may permit selective accumulation of radionuclides administered systemically. AvidinOX is an avidin derivative that resides more persistently than avidin within normal and neoplastic tissues, with a tissue half-life is about two weeks. Its intranipple injection into the ductules of a BALB-neuT mammary gland results in very efficient capture of ${ }^{90} \mathrm{Y}$-biotinDOTA injected intravenously 48 hours later, and leads to the eradication of multifocal cancer lesions [78].

\section{CONCLUSIONS}

The data summarized above show how a dynamic and consistent model of cancer can be exploited to study many features of Her-2 carcinogenesis.

The picture emerging from immunological studies is that even an aggressive mammary carcinogenesis such as that driven by overexpression of an activated transgenic oncogene is modulated by the microenvironment and natural immune surveillance. Maneuvers designed to change a few features of the microenvironment have major consequences on cancer progression. In the BALB-neuT mouse model, modulation of the early stages of tumordriven neoangiogenesis critically affects the pace of mammary carcinogenesis. Vaccines of different kinds provide significant protection against the onset of cancer. Repeated boosting of a vaccine first administered prior to the appearance of a tumor provides lifelong protection. Interventions that temporarily remove suppressor activities may dramatically enhance the ability of a vaccine to cure established cancers.

Lastly, the study of slowly progressing mammary cancer shows the direct and indirect importance of anti-p185 ${ }^{\text {neu }}$ antibodies, whose role is insignificant in models of fast growing transplantable tumors where CTL provides the only therapeutic mechanism. The data from models of autochthonous cancer may serve to change the way antitumor vaccines to be designed, and integrate the induction of antibodies in the immune response among the immune mechanisms to be activated [71, 79].

The consistent progression of mammary lesions of BALB-neuT mice is also exploited to elucidate genes and gene products for their interactions that may modulate ErbB-2-driven carcinogenesis, and exploit new strategy of novel therapeutics.

\section{ACKNOWLEDGMENTS}


The experimental data here reported were supported by grants from Italian Association for Cancer Research (IG 5377, IG 4759); the Italian Ministry for the Universities and Research; the University of Torino; the Compagnia di San Paolo, Torino; and the Regione Piemonte (CIPE 2006 and ImmOnc). This study was funded under the auspices of EUCAAD 200755.

\section{REFERENCES}

[1] Cavallo F, Offringa R, van der Burg SH, Forni G, Melief CJ. Vaccination for treatment and prevention of cancer in animal models. AdvImmunol 2006; 90: 175-213.

[2] Boon T, Cerottini JC, Van den Eynde B, van der Bruggen P, Van Pel A. Tumor antigens recognized by $\mathrm{T}$ lymphocytes. Ann Rev Immunol 1994; 12: 337-65.

[3] Garrido F, Cabrera T, Aptsiauri N. "Hard" and "soft" lesions underlying the HLA class I alterations in cancer cells: implications for immunotherapy. Int J Cancer 2010; 127: 249-56.

[4] Spadaro M, Lanzardo S, Curcio C, Forni G and Cavallo F. Immunological inhibition of carcinogenesis. Cancer Immunol Immunother 2004; 53: 204-16.

[5] Rangarajan A, Weinberg RA. Opinion: Comparative biology of mouse versus human cells: modelling human cancer in mice. Nat Rev Cancer 2003; 3: 952-9.

[6] Lollini P-L, Cavallo F, Nanni P, Forni G. Vaccines for cancer prevention. Nature Rev Cancer 2006; 6: 204-10.

[7] Green JE and Hudson T. The promise of genetically engineered mice for cancer prevention studies. Nature Reviews Cancer 2005; 5: 184-98.

[8] Lin EY, Jones JG, Li P, et al. Progression to malignancy in the polyoma middle $\mathrm{T}$ oncoprotein mouse breast cancer model provides a reliable model for human diseases. Am J Pathol 2003; 163: 2113-26.

[9] Yarden Y, Sliwkowski MX. Untangling the ErbB signalling network.Nat Rev Mol Cell Biol 2001; 2: 127-37.

[10] Hutchinson JN, Muller WJ. Transgenic mouse models of human breast cancer. Oncogene 2000; 19: 6130-7.

[11]Boggio K, Nicoletti G, Di Carlo E, et al. Interleukin 12-mediated prevention of spontaneous mammary adenocarcinomas in two lines of Her-2/neu transgenic mice. J
Exp Med 1998; 188: 589-96.

[12]Lucchini F, Sacco MG, Hu N, et al. Early and multifocal tumors in breast, salivary, harderian and epididymal tissues developed in MMTY-Neu transgenic mice. Cancer Lett 1992; 64: 203-9.

[13] Quaglino E, Mastini C, Forni G, Cavallo F. ErbB2 transgenic mice: a tool for investigation of the immune prevention and treatment of mammary carcinomas. Curr Protoc Immunol Hoboken, NJ, USA: John Wiley \& Sons, Inc. 2008; Chapter 20: Unit 20.9.1-20.9-10.

[14] Pannellini T, Forni G, Musiani P. Immunobiology of Her-2/neu transgenic mice. Breast Dis 2004; 20:33-42.

[15] Diodoro MG, Di Carlo E, Zappacosta R, et al. Salivary carcinoma in HER-2/neu transgenic male mice: an angiogenic switch is not required for tumor onset and progression. Int J Cancer 2000; 88: 329-35.

[16] Pannellini T, Spadaro M, Di Carlo E, et al. Timely DNA vaccine combined with systemic IL-12 prevents parotid carcinomas before a dominant negative p53 makes their growth independent of HER-2/neu expression. J Immunol 2006; 176: 76957703.

[17] Husemann Y, Geigl JB, Schubert F, et al. Systemic spread is an early step in breast cancer. Cancer Cell 2008; 13: 58-68.

[18] Rolla S, Nicolo C, Malinarich S, et al. Distinct and non-overlapping $\mathrm{T}$ cell receptor repertoires expanded by DNA vaccination in wild-type and HER-2 transgenic BALB/c mice. J Immunol 2006; 177: 7626-33.

[19] Rolla S, Ria F, Occhipinti S, et al. Erbb2 DNA vaccine combined with $T$ regulatory cell deletion enhances antibody response and reveals latent low-avidity $\mathrm{T}$ cells. Potential and limits of its therapeutic efficacy. J Immunol 2010; 184: 6124-32.

[20] Melani C, Chiodoni C, Forni G, Colombo MP. Myeloid cell expansion elicited by the progression of spontaneous mammary carcinomas in c-erbB-2 transgenic BALB/c mice suppresses immune reactivity. Blood 2003; 102: 2138-45.

[21] Ambrosino E, Spadaro M, Iezzi M, et al. Immunosurveillance of Erbb2 carcinogenesis in transgenic mice is concealed by a dominant regulatory $\mathrm{T}$-cell self-tolerance. Cancer Res 2006; 66: 773440.

[22] Street SE, Zerafa N, Iezzi M, et al. Host 
perforin reduces tumor number but does not increase survival in oncogene-driven mammary adenocarcinoma. Cancer Res 2007; 67: 5454-60.

[23] Park JM, Terabe M, Donaldson DD, Forni G, Berzofsky JA. Natural immunosurveillance against spontaneous, autochthonous breast cancers revealed and enhanced by blockade of IL-13-mediated negative regulation. Cancer Immunol Immunother 2008; 57: 907 12.

[24] Terabe M, Matsui S, Noben-Trauth N, et al. NKT cell-mediated repression of tumor immunosurveillance by IL-13 and the IL4R-STAT6 pathway. Nat Immunol 2000; 1 : 515-20.

[25] Calogero RA, Cordero F, Forni G, Cavallo F. Inflammatory component of mammary carcinogenesis in Erbb2 transgenic mice. Breast Cancer Res 2007; 9: 211-7.

[26] Chin K, Devries S, Fridlyand J, et al. Genomic and transcriptional aberrations linked to breast cancer pathophiologies. Cancer Cell 2006; 10: 529-41.

[27] Balkwill F. TNF-alpha in promotion and progression of cancer. Cancer Metastasis Rev 2006; 25: 409-16.

[28] Sangaletti S, Tripodo C, Ratti C, et al. Oncogene-driven intrinsic inflammation induces leukocyte production of tumor necrosis factor that critically contributes to mammary carcinogenesis. Cancer Res 2010; 70: 7764-75.

[29] Disis ML, Calenoff E, McLaughlin G, et al. Existent T-cell and antibody immunity to HER-2/neu protein in patients with breast cancer. Cancer Res 1994; 54: 16-29.

[30] Disis ML, Knutson KL, Schiffman K, Rinn $\mathrm{K}, \mathrm{McNeel} \mathrm{DG}$. Pre-existent immunity to the HER-2/neu oncogenic protein in patients with HER-2/neu overexpressing breast and ovarian cancer. Breast Cancer Res Treat 2000; 62: 245-52.

[31]Boggio K, Di Carlo E, Rovero S, et al. Ability of systemic interleukin-12 to hamper progressive stages of mammary carcinogenesis in HER2/neu transgenic mice. Cancer Res 2000; 60: 359-64.

[32] Cifaldi L, Quaglino E, Di Carlo E, et al. A light, nontoxic interleukin 12 protocol inhibits HER-2/neu mammary carcinogenesis in $\mathrm{BALB} / \mathrm{c}$ transgenic mice with established hyperplasia. Cancer Res 2001; 61: 2809-12.

[33] Mastini C, Becker PD, Iezzi M, et al.
Intramammary application of nonmethylated-CpG oligodeoxynucleotides (CpG) inhibits both local and systemic mammary carcinogenesis in female BALB/c Her-2/neu transgenic mice. Current Cancer Drug Targets 2008; 8: 230-42.

[34]Zhang H, Wang Q, Montone KT, et al. Shared antigenic epitopes and pathological functions of anti-p185 (her2/neu) monoclonal antibodies. Exp Mol Pathol 1999; 67: 15-65.

[35] Sharma S, Dominguez AL, Manrique SZ, Cavallo F, Sakaguchi S, Lustgarten J. Systemic targeting of CpG-ODN to the tumor microenvironment with anti-neu-CpG hybrid molecule and $\mathrm{T}$ regulatory cell depletion induces memory responses in BALB-neuT tolerant mice. Cancer Res 2008; 68: 7530-40.

[36] Coscia M, Quaglino E, Iezzi M, et al. Zoledronic acid repolarizes tumorassociated macrophages and inhibits mammary carcinogenesis by targeting the mevalonate pathway. J Cell Mol Med 2010; 14: 2803-15.

[37] Spadaro M, Curcio C, Varadhachary A, et al. Requirement for IFN- $\gamma, \mathrm{CD}^{+} \mathrm{T}$ lymphocytes and NKT cells in Talactoferrininduced inhibition of neutumors. Cancer Res 2007; 67:6425-32.

[38] Rovero S, Amici A, Carlo ED, et al. DNA vaccination against rat her-2/Neu p185 more effectively inhibits carcinogenesis than transplantable carcinomas in transgenic BALB/c mice. J Immunol 2000; 165: 5133 42.

[39] Quaglino E, Mastini C, Iezzi M, et al. The adjuvant activity of BAT antibody enables DNA vaccination to inhibit the progression of established autochthonous Her-2/neu carcinomas in BALB/c mice. Vaccine 2005; 23: 3280-7.

[40] Rovero S, Boggio K, Carlo ED, et al. Insertion of the DNA for the 163-171 peptide of IL1beta enables a DNA vaccine encoding p185(neu) to inhibit mammary carcinogenesis in Her-2/neu transgenic BALB/c mice. Gene Ther 2001; 8: 447-52.

[41] Cappello P, Triebel F, Iezzi M, et al. LAG-3 enables DNA vaccination to persistently prevent mammary carcinogenesis in HER2/neu transgenic BALB/c mice. Cancer Res 2003; 63: 2518-25.

[42] Sloots A, Mastini C, Rohrbach F, et al. DNA vaccines targeting tumor antigens to antigen 
presenting cells in vivo induce protective anti-tumor immunity and delay onset of Her2/neu-driven mammary carcinoma. Clin Cancer Res 2008, 14: 6933-43.

[43] Quaglino E, Iezzi M, Mastini C, et al. Electroporated DNA vaccine clears away multifocal mammary carcinomas in Her2/neu transgenic mice. Cancer Res 2004; 64: 2858-64.

[44] Curcio C, Khan AS, Amici A, et al. DNA immunization using constant-current electroporation affords long-term protection from autochthonous mammary carcinomas in cancer prone transgenic mice. Cancer Gene Ther 2008; 15: 108-14.

[45] Spadaro M, Ambrosino E, Iezzi M, et al. Cure of mammary carcinomas in Her-2 transgenic mice through sequential stimulation of innate (neo-adjuvant il12) and adaptive (DNA Vaccine Electroporation) immunity. Clin Cancer Res 2005; 11: 194152.

[46] Rolla S, Marchini C, Malinarich S, et al. Protective immunity against neu ${ }^{+}$ carcinomas elicited by electroporation of plasmids coding for decreasing fragments of rat neu extracellular domain. Human Gene Ther 2008; 19: 229-40.

[47] Quaglino E, Mastini C, Amici A, et al. A better immune reaction to Erbb-2 tumors is elicited in mice by DNA vaccines encoding rat/human chimeric proteins. Cancer Res 2010; 70: 2604-12.

[48] Dharmapuri S, Aurisicchio L, Neuner P, Verdirame M, Ciliberto G, La Monica N. An oral TLR7 agonist is a potent adjuvant of DNA vaccination in transgenic mouse tumor models. Cancer Gene Ther 2009; 16: 462-72.

[49] Aurisicchio L, Peruzzi D, Conforti A, et al. Treatment of mammary carcinomas in HER2 transgenic mice through combination of genetic vaccine and an agonist of Toll-like receptor 9.Clin Cancer Res. 2009; 15: 1575 84.

[50] Nanni P, Nicoletti G, De Giovanni C, et al. Combined allogeneic tumor cell vaccination and systemic interleukin 12 prevents mammary carcinogenesis in HER-2/neu transgenic mice. J Exp Med 2001; 194: 1195-205.

[51] De Giovanni C, Nicoletti G, Landuzzi L, et al. Immunoprevention of HER-2/neu transgenic mammary carcinoma through an interleukin 12-engineered allogeneic cell vaccine. Cancer Res 2004; 64: 4001-9.
[52] De Giovanni C, Nicoletti G, Palladini A, et al. A multi-DNA preventive vaccine for p53/Neu-driven cancer syndrome. Hum Gene Ther 2009; 20:453-64.

[53] Sakai Y, Morrison BJ, Burke JD, et al. Vaccination by genetically modified dendritic cells expressing a truncated neu oncogene prevents development of breast cancer in transgenic mice. Cancer Res 2004; 64: 8022-8.

[54] Masuelli L, Focaccetti C, Cereda V, et al. Gene-specific inhibition of breast carcinoma in BALB-neuT mice by active immunization with rat Neu or human ErbB receptors. Int J Oncol 2007; 30: 381-92.

[55]Allen SD, Garrett JT, Rawale SV, et al. Peptide vaccines of the Her-2/neu dimerization loop are effective in inhibiting mammary tumor growth in vivo. J Immunol 2007; 179: 472-82.

[56] Garrett JT, Rawale S, Allen, SD, et al. Novel engineered trastuzumab conformational epitopes demonstrate in vitro and in vivo antitumor properties against HER-2/neu. J Immunol 2007; 178: 7120-31.

[57] Nava-Parada P, Forni G, Knutson K L, Pease L R, Celis E. Peptide vaccine given with a Toll-like receptor agonist is effective for the treatment and prevention of spontaneous breast tumors. Cancer Res 2007; 67: 132634.

[58] Tegerstedt K, Lindencrona JA, Curcio C, et al. A single vaccination with polyomavirus VP1/VP2Her2 virus-like particles (VLPs) prevents outgrowth of Her2/neu expressing tumors. Cancer Res 2005; 65: 5953-57.

[59] Masuelli L, Marzocchella L, Focaccetti C, et al. Local delivery of recombinant vaccinia virus encoding for neu counteracts growth of mammary tumors more efficiently than systemic delivery in neu transgenic mice.Cancer Immunol Immunother 2010; 59: 1247-58.

[60] Park JM, Terabe M, Sakai Y, et al. Early Role of $\mathrm{CD}^{+}$Th1 cells and antibodies in HER-2 adenovirus-vaccine protection against autochthonous mammary carcinomas. J Immunol 2005; 174: 4228-36.

[61] Gallo P, Dharmapuri S, Nuzzo M, et al. Adenovirus vaccination against neu oncogene exerts long-term protection from tumorigenesis in BALB/neuT transgenic mice. Int J Cancer 2007; 120: 574-84.

[62] Cipriani B, Fridman A, Bendtsen C, et al. Therapeutic vaccination halts disease 
progression in BALB-neuT mice: the amplitude of elicited immune response is predictive of vaccine efficacy. Hum Gene Ther 2008; 19: 670-80.

[63] Quaglino E, Rolla S, Iezzi M, et al. Concordant morphologic and gene expression data show that a vaccine halts HER-2/neupreneoplastic lesions. J Clin Invest 2004; 113: 709-17.

[64] Nanni P, Landuzzi L, Nicoletti G, et al. Immunoprevention of mammary carcinoma in Her-2/neu transgenic mice is $\gamma$-interferon and B cell dependent. J Immunol 2004; 173 : 2288-96.

[65] Porzia A, Lanzardo S, Citti A, et al.Attenuation of PI3K/AKT-mediated tumorigenic signals through PTEN activation by DNA vaccine-induced antiErbB2 antibodies. J Immunol 2010; 184: 4170-7.

[66] Astolfi A, Landuzzi L, Nicoletti G, et al. Gene expression analysis of immunemediated arrest of tumorigenesis in a transgenic mouse model of HER-2/neupositive basal-like mammary carcinoma. Am J Pathol 2005; 166: 1205-16.

[67] Astolfi A, Rolla S, Nanni P, et al. Immune prevention of mammary carcinogenesis in HER-2/neu transgenic mice: a microarray scenario. Cancer Immunol Immunother 2005; 54: 599-610.

[68] Holmgren L, Ambrosino E, Birot O, et al. A DNA vaccine targeting angiomotin inhibits angiogenesis and suppresses tumor growth. Proc Natl Acad Sci USA 2006; 103: 920813.

[69] Cavallo F, Astolfi A, Iezzi M, et al. An integrated approach of immunogenomics and bioinformatics to identify new Tumor Associated Antigens (TAA) for mammary cancer immunological prevention. BMC Bionformatics 2005; 6 (S4): 1-9.

[70] Calogero RA, Quaglino E, Saviozzi S, Forni $\mathrm{G}$, and Cavallo F. Oncoantigens as antitumor vaccination targets: the chance of a lucky strike? Cancer Immunol Immunother 2008; 57: 1685-94.

[71] Cavallo F, Calogero RA, Forni G. Are oncoantigens suitable targets for antitumor therapy? Nature Rev Cancer 2007; 7: 70712.

[72] Cabodi S, Tinnirello A, Di Stefano P, et al. p130Cas as a new regulator of mammary gland development and of HER2-Neudependent tumorigenesis. Cancer Res 2006;
66: 4672-80.

[73] Cabodi S, Tinnirello A, Bisaro B, et al. p130Cas is an essential transducer element in ErbB2 transformation. FASEB J 2010; 24 : 3796-80.

[74] Barbieri I, Quaglino E, Maritano D, et al. Stat3 is required for anchorage-independent growth and metastasis but not for mammary tumor development downstream of the ErbB-2 oncogene. Molecular Carcinogenesis 2010; 49: 114-20.

[75] Barbieri I, Pensa S, Pannellini T, et al. Constitutively active Stat3 enhances neumediated migration and metastasis in mammary tumors via upregulation of Cten. Cancer Res 2010; 70 : 2558-67.

[76] Ciraolo E, Curcio C, Marone R, et al. Key role of phosphoinositide 3-Kinase p110beta catalytic function in metabolism and cancer but not development. Science Signalling 2008; 1: 1-12.

[77] Schramek D, Kotsinas A, Meixner A, et al. The stress kinase MKK7 couples oncogenic stress to p53 stability and tumor suppression. Nat Genet 2011; 43: 212-9.

[78]De Santis R, Leoni B, Rosi A, et al. AvidinOX $\mathrm{X}^{\mathrm{TM}}$ for avidin for highly efficient tissue pre-targeted radionuclide therapy. Cancer Biotherapy \& Radiopharmaceuticals 2010; 25: 143-8.

[79] Cavallo F, De Giovanni C, Nanni P, Forni G, Lollini PL. 2011: the immune hallmarks of cancer. Cancer Immunol Immunother 2011; 60: 319-26. 